\title{
Antagonistic and Detoxification Potentials of Trichoderma Isolates for Control of Zearalenone (ZEN) Producing Fusarium graminearum
}

\author{
Ye Tian ${ }^{1}$, Yanglan Tan ${ }^{1}$, Zheng Yan ${ }^{1}$, Yucai Liao ${ }^{2}$, Jie Chen ${ }^{3}$, Marthe De Boevre ${ }^{4}$, \\ Sarah De Saeger ${ }^{4}$ and Aibo Wu ${ }^{1 *}$ \\ ${ }^{1}$ SIBS-UGENT-SJTU Joint Laboratory of Mycotoxin Research, Key Laboratory of Food Safety Research, Shanghai Institutes \\ for Biological Sciences, University of Chinese Academy of Sciences, Chinese Academy of Sciences, Shanghai, China, \\ ${ }^{2}$ College of Plant Science and Technology, Huazhong Agricultural University, Wuhan, China, ${ }^{3}$ Department of Resources and \\ Environment Sciences, School of Agriculture and Biology, Shanghai Jiaotong University, Shanghai, China, ${ }^{4}$ Laboratory of \\ Food Analysis, Department of Bioanalysis, Faculty of Pharmaceutical Sciences, Ghent University, Ghent, Belgium
}

OPEN ACCESS

Edited by:

Mehdi Razzaghi-Abyaneh,

Pasteur Institute of Iran, Iran

Reviewed by:

Sabrina Sarrocco,

University of Pisa, Italy

Michelina Ruocco,

Istituto per la Protezione Sostenibile

delle Piante (CNR), Italy

*Correspondence:

Aibo Wu

abwu@sibs.ac.cn

Specialty section:

This article was submitted to

Food Microbiology,

a section of the journal

Frontiers in Microbiology

Received: 29 October 2017 Accepted: 29 December 2017

Published: 18 January 2018

Citation:

Tian Y, Tan Y, Yan Z, Liao Y, Chen J, De Boevre M, De Saeger S and WU A (2018) Antagonistic and Detoxification Potentials of Trichoderma Isolates for

Control of Zearalenone (ZEN)

Producing Fusarium graminearum.

Front. Microbiol. 8:2710.

doi: 10.3389/fmicb.2017.02710
Fungi belonging to Fusarium genus can infect crops in the field and cause subsequent mycotoxin contamination, which leads to yield and quality losses of agricultural commodities. The mycotoxin zearalenone (ZEN) produced by several Fusarium species (such as F. graminearum and F. culmorum) is a commonly-detected contaminant in foodstuffs, posing a tremendous risk to food safety. Thus, different strategies have been studied to manage toxigenic pathogens and mycotoxin contamination. In recent years, biological control of toxigenic fungi is emerging as an environment-friendly strategy, while Trichoderma is a fungal genus with great antagonistic potentials for controlling mycotoxin producing pathogens. The primary objective of this study was to explore the potentials of selected Trichoderma isolates on ZEN-producing F. graminearum, and the second aim was to investigate the metabolic activity of different Trichoderma isolates on ZEN. Three tested Trichoderma isolates were proved to be potential candidates for control of ZEN producers. In addition, we reported the capacity of Trichoderma to convert ZEN into its reduced and sulfated forms for the first time, and provided evidences that the tested Trichoderma could not detoxify ZEN via glycosylation. This provides more insight in the interaction between ZEN-producing fungi and Trichoderma isolates.

Keywords: mycotoxins, zearalenone (ZEN), Fusarium, biological control, Trichoderma, modified mycotoxins

\section{INTRODUCTION}

Mycotoxins are secondary metabolites produced by fungi with toxic effects on plants, animals and human (Hussein and Brasel, 2001). Among them, zearalenone (ZEN) is a mycotoxin with estrogenic potency, and commonly found in agricultural commodities globally (Nielsen et al., 2014). ZEN is mainly synthesized by a variety of Fusarium species, such as F. graminearum, F. culmorum, and F. crookwellense, which are plant pathogens capable of infecting crops and causing crop diseases in the field (Zinedine et al., 2007; Gromadzka et al., 2008). Besides ZEN, zearalanone (ZAN), $\alpha$-zearalenol ( $\alpha$-ZOL), $\beta$-zearalenol $(\beta$-ZOL), $\alpha$-zearalanol $(\alpha-Z A L)$, and $\beta$-zearalanol ( $\beta$-ZAL) (Figure 1) are reduced derivatives of ZEN frequently detected in contaminated cereal grains or cultures of ZEN-producing Fusarium species (Zinedine et al., 2007). 
As toxic xenobiotics for plants, ZEN, $\alpha$-ZOL, and $\beta$-ZOL can be bio-transformed into less toxic ZEN-14-glucoside (Z14G), $\alpha$-ZOL-14-glucoside $(\alpha$-ZOL14G) and $\beta$-ZOL-14-glucoside $(\beta$ ZOL14G) (Figure 1), which are common detoxification products in plant defense. Furthermore, some fungi also possess the capacity to convert ZEN into Z14G by conjugating endogenous glucose (Kamimura, 1986). The structures of these glycosylated mycotoxins were changed after modification, so they can't be detected by routine analysis, and they are termed as modified mycotoxins (Berthiller et al., 2013).

Both ZEN and its reduced forms are stable compounds, which exhibit hepatotoxic, hematotoxic, immunotoxic, and genotoxic effects. In addition, ZEN-related mycotoxins can competitively bind the estrogen receptors, causing alterations in genitals and reproduction disorders, posing a threat to human and animals health (Zinedine et al., 2007). To protect consumers, prevention before harvest seems to be an effective strategy for mycotoxin management (Jard et al., 2011; Tian et al., 2016a). Application of antagonistic biological control agents for controlling the toxigenic Fusarium spp., is a promising biological control based approach to reduce ZEN contamination. As potential antagonistic microbes, the genus Trichoderma has been widely studied for their capabilities against plant pathogenic fungi, and its biological control mechanisms mainly include faster growth speed and antibiotic production to compete nutrients and living space with pathogens, mycoparasitism mediated by producing cell wall degrading enzymes, and the ability to induce plant's defense system (Benítez et al., 2004; Sellamani et al., 2016; Tian et al., 2016a). Two Trichoderma isolates could effectively decrease the amount of mycotoxin ZEN produced by Fusarium spp. by a dual-culture assay (Gromadzka et al., 2009). In addition, other studies showed that some Trichoderma isolates also could inhibit mycotoxin deoxynivalenol (DON) production of Fusarium spp. (Busko et al., 2008; Matarese et al., 2012; Tian et al., 2016b). DON, a common type B trichothecene mycotoxin (Cuomo et al., 2007), usually co-occurs with ZEN in the foods and feeds (Molto et al., 1997; Castillo et al., 2002; Döll and Dänicke, 2011; Pietsch et al., 2013; Kovalsky Paris et al., 2014). Both DON and ZEN are frequently detected mycotoxins with high contamination levels (Stepien and Chełkowski, 2010). Recent work showed that DON could be bio-transformed into its modified form deoxynivalenol-3-glucoside (D3G) by Trichoderma isolates. D3G was generated in the defense of plants after infected by DON-producing pathogens, and D3G was regarded as a detoxification product of DON catalyzed by UDP-glucosyltransferases (Poppenberger et al., 2003; Schweiger et al., 2010; Li et al., 2015; Pasquet et al., 2016). Our recent study reported the occurrence of D3G in metabolism of selected Trichoderma isolates against DON producers (Tian et al., 2016b). However, little is known about the metabolism of ZEN in Trichoderma isolates. Thus, our particular interest was that whether Trichoderma spp. also possess the capacity to glycosylate ZEN into glycosylated forms for self-protection. Herein, the antagonistic potentials of Trichoderma isolates against ZENproducing $F$. graminearum and the metabolism of $\mathrm{ZEN}$ in Trichoderma isolates was investigated in this work. A targeted LC-MS/MS method for simultaneous determination of ZEN and its reduced forms $(\alpha-Z O L, \beta-Z O L, \alpha-Z A L, \beta-Z A L$, and ZAN) and glycosylated forms (Z14G, $\alpha$-ZOL14G, and $\beta-Z O L 14 G)$ was applied to explore the anti-toxigenic activity of antagonists and ZEN metabolization in Trichoderma isolates. It was observed that three Trichoderma isolates could effectively suppress the mycelia spread and mycotoxin production of ZEN-producing F. graminearum. In addition, results of ZEN-treated experiment showed that the tested Trichoderma isolates could not detoxify ZEN via glycosylation, but could convert ZEN to its reduced ( $\alpha$ ZOL and $\beta-Z O L)$ and sulfated metabolites (Z14S and ZOL14S). As far as we know, this is the first report of the metabolic activity of Trichoderma isolates on ZEN, which would provide more insights in the interaction between mycotoxin ZEN producing fungi and antagonistic Trichoderma isolates.

\section{MATERIALS AND METHODS}

\section{Chemicals and Reagents}

The mycotoxin standards of ZEN, $\alpha$-ZOL, $\beta$-ZOL, $\alpha$-ZAL, $\beta$ $Z A L$, and ZAN were purchased from Sigma-Aldrich (St. Louis, MO, USA). The standards of Z14G, $\alpha$-ZOL14G, and $\beta-Z O L 14 G$ were kindly provided by the Laboratory of Food Analysis, Ghent University (Belgium). Methanol and acetonitrile (HPLC-grade) were purchased from Merck (Darmstadt, Germany). Ultrapure water $(18.2 \mathrm{M} \Omega \cdot \mathrm{cm})$ used in our experiments was obtained from a Milli-Q System (Bedford, MA, USA). Cleanert MC cleanup columns were purchased from Bonna-Agela Technologies (Tianjin, China). Other chemicals were obtained from Aladdin (Shanghai, China).

\section{Fungal Isolates}

ZEN-producing F. graminearum species were from the Huazhong Agricultural University. Eight Trichoderma isolates were used in this study: T. harzianum JF309, T. koningii GIM3.137, T. harzianum GIM3.442, T. longibranchiatum GIM3.534, T. harzianum Q710613, T. atroviride Q710251, T. asperellum Q710682 and T. virens Q710925. All these Trichoderma isolates could convert DON into D3G, as reported in our previous work (Tian et al., 2016b).

\section{Antagonistic Potentials of Trichoderma Isolates on Growth and Mycotoxin Production of $F$. graminearum $F 1$}

The dual-culture test was performed to examine the antagonistic potentials of Trichoderma isolates for controlling ZENproducing F. graminearum as described before (Matarese et al., 2012; Tian et al., 2016b). The mycelial disks (F. graminearum and Trichoderma spp. combinations) from actively-growing colonies were placed on a 9-cm diameter dish. In addition, a disk of F. graminearum was placed without disks of Trichoderma isolates (control). The Fusarium-Trichoderma combinations, as well as the controls were incubated at $25^{\circ} \mathrm{C}$ for 2 weeks. To evaluate the inhibition efficacy of Trichoderma isolates on mycelia growth of $F$. graminearum $\mathrm{F} 1$, the radius of each $F$. graminearum colony was measured to create growth curve as described in Matarese et al. (2012), and then the data were subjected to analysis of variance of regression to compare the slope of growth curves 


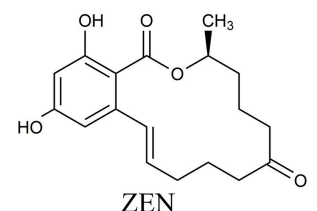

ZEN<smiles>Cc1cc(O)cc(O)c1C(=O)OC(C)CCCC(=O)CCCCC#N</smiles>

ZAN

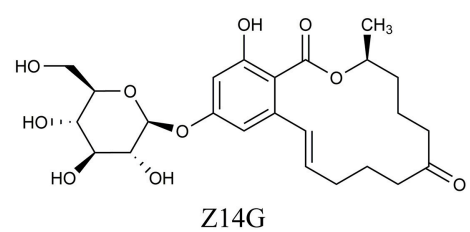

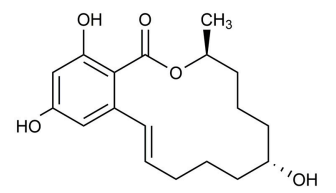

$\alpha-\mathrm{ZOL}$
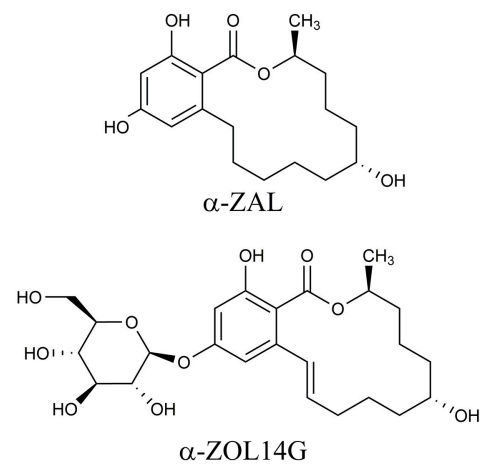<smiles>C[C@@H](CCCC(O)CC/C=C/c1cc(O)cc(O)c1C(=O)O)OC(=O)[O-]</smiles>

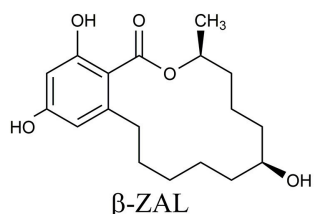

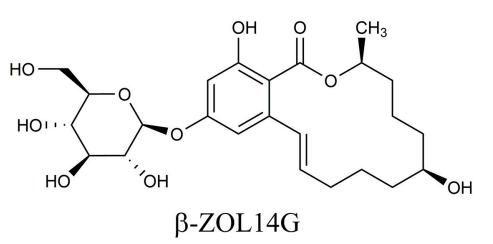

FIGURE 1 | Chemical structures of ZEN, $\alpha$-ZOL, $\beta$-ZOL, ZAN, $\alpha$-ZAL, $\beta$-ZAL, Z14G, $\alpha$-ZOL14G, and $\beta$-ZOL14G.

of the pathogen in the presence/absence of tested Trichoderma isolates.

\section{Treatment of Trichoderma Isolates with ZEN}

The tested Trichoderma isolates were activated on PDA medium at $25^{\circ} \mathrm{C}$. Then, a mycelial disk of each activated Trichoderma strain was moved from the edge of the colony, and inoculated in a new dish containing of $10 \mathrm{ml}$ PDA medium mixed with ZEN at different concentrations $(0,0.5,1,2$, and $4 \mu \mathrm{g} / \mathrm{ml})$. Pure ZEN was added into the PDA medium as described before (Utermark and Karlovsky, 2007). The mycelial disk of Trichoderma isolates placed on PDA medium without mycotoxin was as control. The dishes were incubated at $25^{\circ} \mathrm{C}$, and growth radius of the tested Trichoderma isolates were measured two times a day until the mycelia of tested strains spread over the whole dish. Regression analysis of the growth data was performed to evaluate the inhibitory effects of ZEN on mycelial growth of Trichoderma isolates.

\section{Mycotoxin Extraction}

After incubation, the PDA medium and mycelia in the dish were dried and ground into powder for preparation, followed by adding $10 \mathrm{ml}$ of ACN/water/formic acid (84/15.9/0.1, v/v/v) solution. The mixture was then shaken for $10 \mathrm{~min}$, and subjected to ultrasonication for $30 \mathrm{~min}$. Next the mixture was centrifuged at $4,000 \mathrm{rpm}$ for $30 \mathrm{~min}$. $1 \mathrm{ml}$ of the supernatant was passed through a Cleanert MC column for clean-up by following the manufacturer instructions. Thereafter, the purified mixture was moved into a new tube, and evaporated to dryness by nitrogen gas at $45^{\circ} \mathrm{C}$. Finally, the residue was re-dissolved with $1 \mathrm{~mL}$ of methanol/water $(1 / 1, \mathrm{v} / \mathrm{v})$ and filtered through a $0.22-\mu \mathrm{m}$ filter for LC-MS/MS analysis.

\section{Mycotoxin Analysis by LC-MS/MS}

Mycotoxins were determined on an Accela 1250 UPLC system (Thermo Fisher Scientific, San Jose, CA, USA) coupled to a TSQ VantageTM (Thermo Fisher Scientific, San Jose, CA, USA) triple stage quadrupole mass spectrometer. Separation was performed on an Agilent Extend-C18 column $(100 \mathrm{~mm} \times 4.6 \mathrm{~mm}, 3.5 \mu \mathrm{m})$ at $30^{\circ} \mathrm{C}$ with a flow rate of $350 \mu \mathrm{L} / \mathrm{min}$. The mobile phase consisted of water containing $5 \mathrm{mM}$ ammonium acetate (A) and methanol (B). The gradient was as follows: $0 \mathrm{~min} 20 \% \mathrm{~B}, 1 \mathrm{~min}$ $20 \%$ B, 2 min $50 \%$ B, 8 min $100 \%$ B, 10 min $100 \%$ B, 13 min $20 \%$ $\mathrm{B}, 15 \mathrm{~min} 20 \% \mathrm{~B}$. The injection volume was $10 \mu \mathrm{L}$.

For MS/MS analysis, the parameters were set as follows: interface voltage of $2.5 \mathrm{kV}\left(\mathrm{ESI}^{-}\right)$; desolvation temperature of $270^{\circ} \mathrm{C}$; nebulizing gas $\left(\mathrm{N}_{2}\right)$ pressure of 50 psi and drying gas $\left(\mathrm{N}_{2}\right)$ pressure of 25 psi; heat block temperature of $300^{\circ} \mathrm{C}$. The quantitation and identification of target mycotoxins were performed in selected reaction monitoring (SRM) mode. The optimized MS/MS parameters for each analyte are listed in Table 1. Xcalibur ${ }^{\mathrm{TM}}$ software (Thermo Fisher Scientific, San Jose, CA, USA, 2011) was used for data processing.

\section{Liquid Chromatography High Resolution Mass Spectrometry (LC-HRMS) Analysis of ZEN Bio-transformation Products}

LC-HRMS analysis was conducted on a UHPLC system (1290 series, Agilent Technologies, Santa Clara, CA, USA) coupled to a quadruple time-of-flight (Q-TOF) mass spectrometer (Agilent 6530 Q-TOF, Agilent Technologies, Santa Clara, CA, USA). Chromatographic separation was performed on the Agilent Extend-C18 column. The mobile phase consisted of water containing $5 \mathrm{mM}$ ammonium acetate (A) and methanol (B), and the gradient elution program was: $0 \mathrm{~min} 20 \% \mathrm{~B}, 1 \mathrm{~min} 20 \% \mathrm{~B}$, $2 \min 50 \% \mathrm{~B}, 8 \mathrm{~min} 100 \% \mathrm{~B}, 10 \mathrm{~min} 100 \% \mathrm{~B}, 13 \mathrm{~min} 20 \% \mathrm{~B}$, $15 \min 20 \% \mathrm{~B}$. 
TABLE 1 | MS/MS parameters for detected mycotoxins in SRM mode.

\begin{tabular}{|c|c|c|c|c|c|c|}
\hline Mycotoxin & Precursor ion $(\mathrm{m} / \mathrm{z})$ & $\begin{array}{l}\text { Retention } \\
\text { time (min) }\end{array}$ & $\begin{array}{c}\text { Product ion for } \\
\text { quantification }(\mathrm{m} / \mathrm{z})\end{array}$ & $\begin{array}{c}\text { Collision } \\
\text { energy (ev) }\end{array}$ & $\begin{array}{c}\text { Product ion for } \\
\text { identification }(\mathrm{m} / \mathrm{z})\end{array}$ & $\begin{array}{l}\text { Collision } \\
\text { energy (ex }\end{array}$ \\
\hline ZEN & $317.2[\mathrm{M}-\mathrm{H}]^{-}$ & 8.60 & 175.5 & 26 & 131.5 & 32 \\
\hline ZAN & $319.1[\mathrm{M}-\mathrm{H}]^{-}$ & 8.44 & 205.5 & 23 & 160.5 & 24 \\
\hline$\alpha-Z O L$ & $319.3[\mathrm{M}-\mathrm{H}]^{-}$ & 8.29 & 160.5 & 22 & 130.5 & 33 \\
\hline$\beta$-ZOL & $319.3[\mathrm{M}-\mathrm{H}]^{-}$ & 7.73 & 160.5 & 22 & 130.5 & 33 \\
\hline$\alpha-Z A L$ & $321.3[\mathrm{M}-\mathrm{H}]^{-}$ & 8.11 & 277.5 & 24 & 303.5 & 23 \\
\hline$\beta-Z A L$ & $321.3[\mathrm{M}-\mathrm{H}]^{-}$ & 7.45 & 277.5 & 23 & 303.5 & 24 \\
\hline Z14G & $479.0[\mathrm{M}-\mathrm{H}]^{-}$ & 6.78 & 317.5 & 20 & 175.6 & 45 \\
\hline$\alpha-Z O L 14 G$ & $481.0[\mathrm{M}-\mathrm{H}]^{-}$ & 6.61 & 319.6 & 18 & 275.5 & 37 \\
\hline$\beta$-ZOL14G & $481.0[\mathrm{M}-\mathrm{H}]^{-}$ & 5.78 & 319.6 & 23 & 275.5 & 36 \\
\hline
\end{tabular}

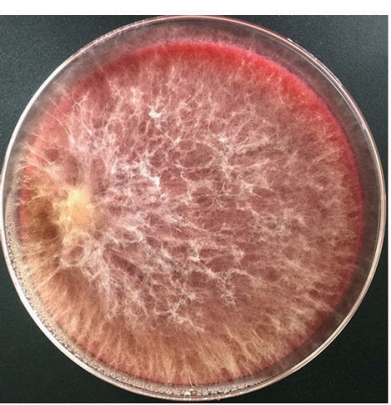

A

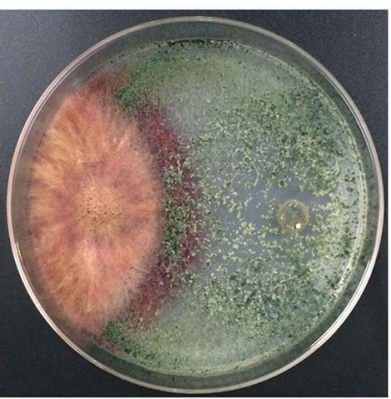

D

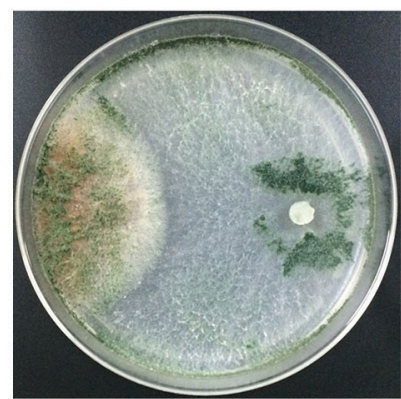

G

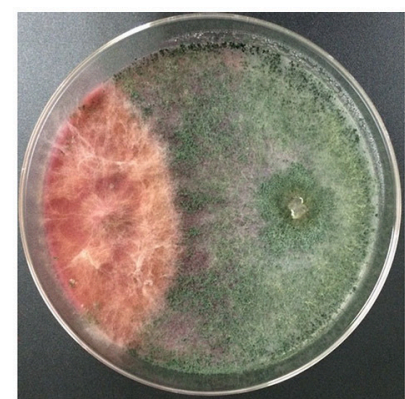

B

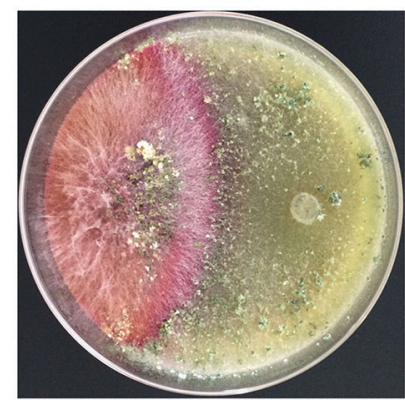

E

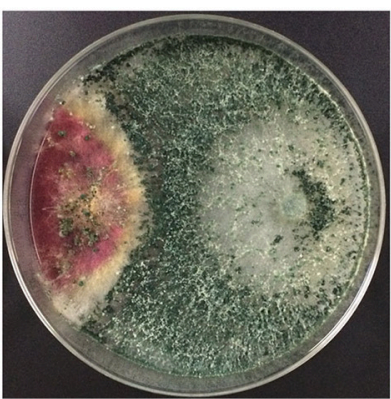

H



C

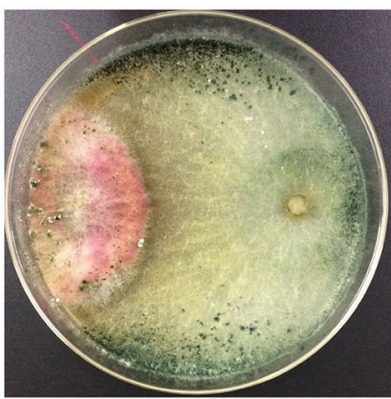

$\mathbf{F}$

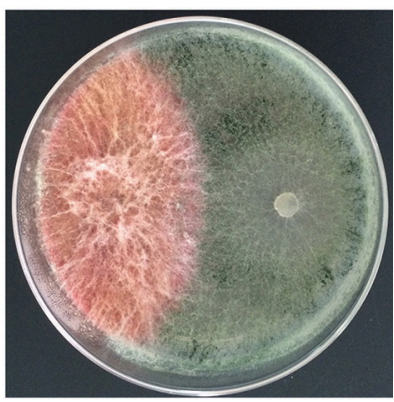

I

FIGURE 2 | Colony morphology of F. graminearum F1 in co-culture assay after incubation on the potato dextrose agar (PDA). F. graminearum F1 grew alone (A); F. graminearum 5035 grew against T. harzianum JF309 (B); T. koningii GIM3.137 (C); T. harzianum GIM3.442 (D); T. Iongibranchiatum GIM3.534 (E); T. harzianum Q710613 (F); T. atroviride Q710251 (G); T. asperellum Q710682 (H); and T. virens Q710925 (I). 
The parameters of HRMS were set as follows: sheath gas (N2) temperature, $350^{\circ} \mathrm{C}$, sheath gas flow, $11 \mathrm{~L} / \mathrm{min}$; dry gas $(\mathrm{N} 2)$ temperature, $350^{\circ} \mathrm{C}$, dry gas flow, $11 \mathrm{~L} / \mathrm{min}$; capillary voltage, $3.8 \mathrm{kV}$ in negative mode; fragmentor, $130 \mathrm{~V}$; and nebulizer pressure, 40 psi. Agilent software MassHunter B.06.00 (Agilent Technologies, Santa Clara, CA, USA, 2012) was used for data processing.

\section{Statistical Analysis}

All the experiments were set up in triplicates. Data were presented as the mean \pm standard error of the mean (SEM), and data were subjected to two-tailed student's $t$-test analysis or regression analysis with Graphpad Prism 5.01 (GraphPad Software, San Diego, CA, USA, 2007).

\section{RESULTS}

\section{Effect of Trichoderma Isolates on Growth of ZEN-Producing $F$. graminearum $\mathrm{F} 1$ in Co-culture Assay}

Trichoderma isolates and F. graminearum F1 were co-cultured on PDA medium. These antagonists rapidly occupied the living space, and inhibited the mycelial spread of $F$. graminearum F1 due to their antagonistic activities (Figure 2). Growth inhibition is the main pattern for antagonists to manage the pathogen, and we found that seven of the tested Trichoderma isolates were able to significantly suppress the mycelial growth of F. graminearum F1 (Table 2). Furthermore, T. harzianum Q710613, T. atroviride Q710251 and T. asperellum Q710682 displayed more effective inhibitory effects, as we observed that these three Trichoderma isolates overgrew the colony of F. graminearum $\mathrm{F} 1$, and the mycelium of Fusarium in these pathogen-antagonist combinations were restricted to extend vertically (Figure 2). These results showed that $T$. harzianum Q710613, T. atroviride Q710251, and T. asperellum Q710682 were more effective suppressors for controlling the mycelia growth of F. graminearum F1.

\section{Effect of Trichoderma Isolates on Mycotoxin Production of ZEN-Producing F. graminearum F1 in Co-culture Assay}

To investigate the effect of Trichoderma isolates on ZEN-related mycotoxins production of $F$. graminearum, and verify whether Trichoderma could glycosylate ZEN into glycosylated forms. ZEN and its reduced derivatives $(\alpha-Z O L, \beta-Z O L, \alpha-Z A L, \beta-$ $Z A L$, and ZAN), as well as three glycosylated mycotoxins (Z14G, $\alpha$-ZOL14G, and $\beta$-ZOL14G), were monitored in this work.

$F$. graminearum $\mathrm{F} 1$ used in this dual-culture assay could produce $1562 \mu \mathrm{g} / \mathrm{g} \mathrm{ZEN}, 27 \mu \mathrm{g} / \mathrm{g} \mathrm{ZAN}, 2.6 \mu \mathrm{g} / \mathrm{g} \alpha-\mathrm{ZOL}$, and $15 \mu \mathrm{g} / \mathrm{g} \beta$-ZOL on PDA medium (Figure 3). ZEN was the major mycotoxin produced by the tested F. graminearum. When $F$. graminearum $\mathrm{F} 1$ grew against antagonistic Trichoderma isolates, the amount of mycotoxins produced by F. graminearum F1 was inhibited because of the antagonistic activity of Trichoderma. The inhibition rate of ZEN production ranged
TABLE 2 | The inhibitory effect of antagonistic Trichoderma isolates on mycelial growth of $F$. graminearum $\mathrm{F} 1$ in dual culture.

\begin{tabular}{|c|c|c|c|c|}
\hline \multirow[t]{2}{*}{ Combination } & \multicolumn{4}{|c|}{ Regression parameters of growth curves } \\
\hline & & $a$ & $P$ & P slope \\
\hline \multirow[t]{2}{*}{ FG F1 vs. T. harzianum JF309 } & Gc & 0.49 & $<0.0001$ & $<0.0001$ \\
\hline & Gt & 0.37 & $<0.0001$ & \\
\hline \multirow[t]{2}{*}{ FG F1 vs. T. koningii GIM3.137 } & Gc & 0.49 & $<0.0001$ & NS \\
\hline & Gt & 0.45 & $<0.0001$ & \\
\hline \multirow{2}{*}{$\begin{array}{l}\text { FG F1 vs. T. harzianum } \\
\text { GIM3.442 }\end{array}$} & Gc & 0.49 & $<0.0001$ & $<0.0001$ \\
\hline & Gt & 0.40 & $<0.0001$ & \\
\hline \multirow{2}{*}{$\begin{array}{l}\text { FG F1 vs. T. longibranchiatum } \\
\text { GIM3.534 }\end{array}$} & Gc & 0.49 & $<0.0001$ & $<0.0001$ \\
\hline & Gt & 0.42 & $<0.0001$ & \\
\hline \multirow{2}{*}{$\begin{array}{l}\text { FG F1 vs. T. harzianum } \\
\text { Q710613 }\end{array}$} & Gc & 0.49 & $<0.0001$ & $<0.0001$ \\
\hline & Gt & 0.35 & $<0.0001$ & \\
\hline \multirow[t]{2}{*}{ FG F1 vs. T. atroviride Q710251 } & $\mathrm{Gc}$ & 0.49 & $<0.0001$ & $<0.0001$ \\
\hline & Gt & 0.33 & $<0.0001$ & \\
\hline \multirow{2}{*}{$\begin{array}{l}\text { FG F1 vs. T. asperellum } \\
\text { Q710682 }\end{array}$} & Gc & 0.49 & $<0.0001$ & $<0.0001$ \\
\hline & Gt & 0.32 & $<0.0001$ & \\
\hline \multirow[t]{2}{*}{ FG F1 vs. T. virens Q710925 } & Gc & 0.49 & $<0.0001$ & $<0.0001$ \\
\hline & Gt & 0.40 & $<0.0001$ & \\
\hline
\end{tabular}

The radial growth rate of $F$. graminearum $F 1$ facing antagonists on PDA medium compared with the radial growth rate of the control.

a, slope of the growth curve of F. graminearum (growth rate, $\mathrm{mm} / \mathrm{hour}$ ).

$P$, significance of regression line.

$P$ slope, significance of the difference between slopes of the pathogen F1 in the presence (Gt) and absence (Gc) of tested Trichoderma isolates.

NS, no significant difference.

from 9 to $97 \%$, for ZAN ranged from 22 to $98 \%$, for $\alpha$ ZOL ranged from 31 to $87 \%$, and for $\beta$-ZOL ranged from 34 to $89 \%$. Among the tested isolates, T. koningii GIM3.137 exhibited weaker inhibitory effects on the mycotoxin production of F. graminearum F1 (Figure 3). While T. harzianum Q710613, T. atroviride Q710251, and T. asperellum Q710682 exhibited a better efficiency in inhibiting mycotoxin production of Fusarium. When co-cultured with these three isolates, the amount of ZEN and ZAN produced by $F$. graminearum $F 1$ was inhibited by over $93 \%$, and the amount of $\alpha-Z O L$ and $\beta$-ZOL produced by F. graminearum $\mathrm{F} 1$ was inhibited by over $80 \%$.

Unexpectedly, no glycosylated forms of ZEN and ZOL were observed when Trichoderma isolates were co-cultured with ZEN-producing Fusarium. The experiment, as described below, pinpointed the treatment of Trichoderma isolates with ZEN, and analyzed the metabolites to confirm the obtained result.

\section{Analysis of the Metabolites When Trichoderma Grew on PDA Medium Amended with Pure Mycotoxin ZEN} The Inhibition of ZEN on Growth of Trichoderma

It has been reported that ZEN could inhibit the growth of some filamentous fungi, which help ZEN-producing Fusarium species compete with other microbes, so ZEN is regarded as a contributive factor for ZEN-producers (Utermark and Karlovsky, 2007). Firstly, we evaluated the inhibitory effects of ZEN on growth of various Trichoderma isolates, as the toxic effects of 
A

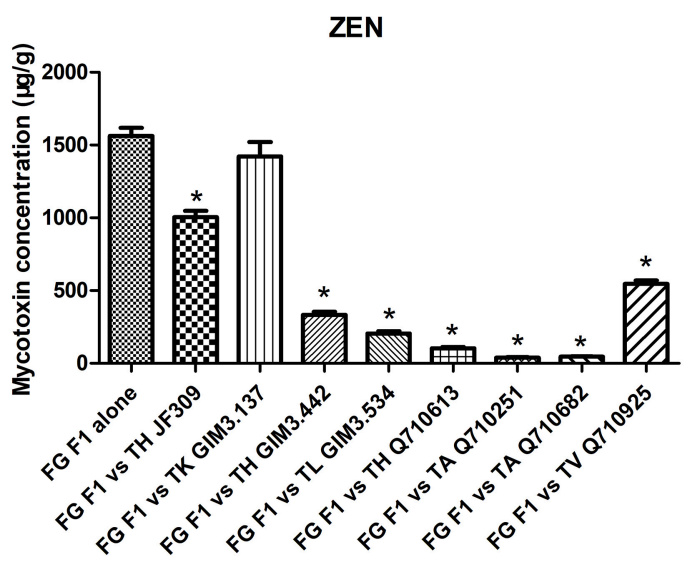

C

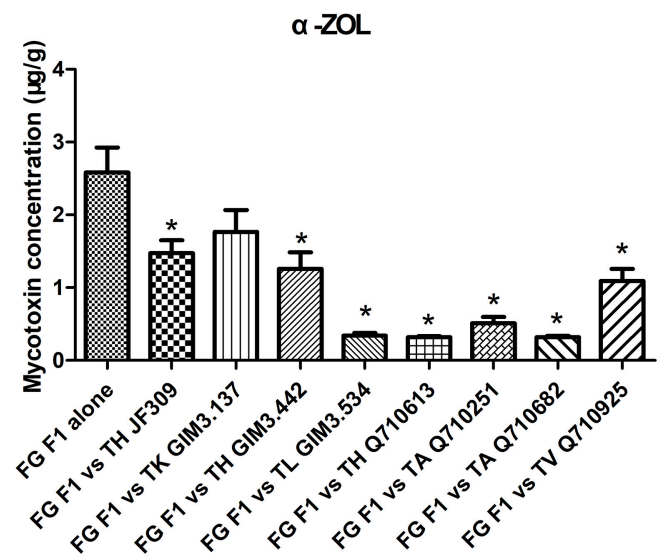

B

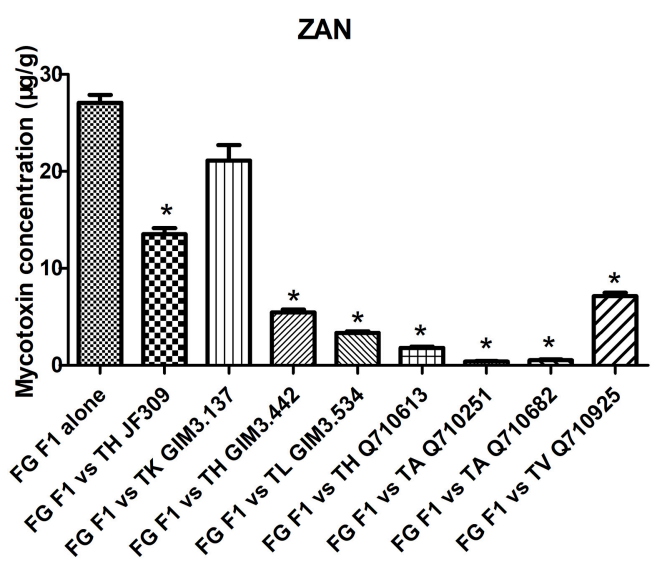

D

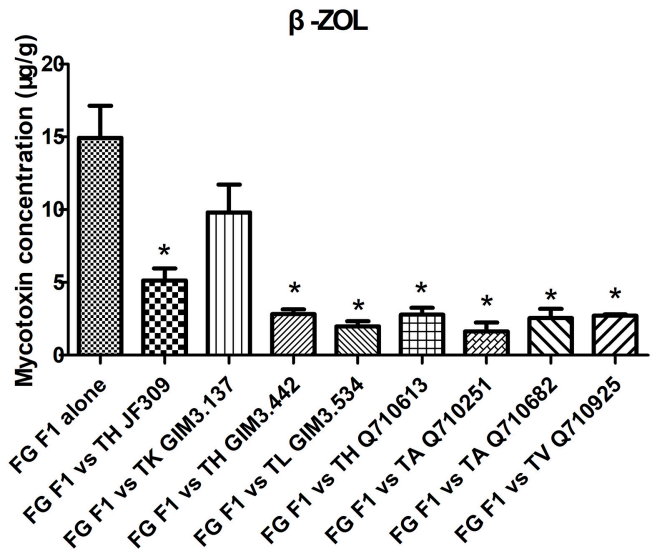

FIGURE 3 | The inhibitory effect of antagonistic Trichoderma isolates on mycotoxin production (ZEN, A; ZAN, B; $\alpha$-ZOL, C; and $\beta$-ZOL, D) of $F$. graminearum F1 in co-culture assay. From left to right: F. graminearum F1 grew alone, and grew against T. harzianum JF309, T. koningii GIM3.137, T. harzianum GIM3.442,

T. Iongibranchiatum GIM3.534, T. harzianum Q710613, T. atroviride Q710251, T. asperellum Q710682, and T. virens Q710925. ${ }^{*} P<0.05$, significantly different from control.

ZEN have not yet been elucidated in the genus Trichoderma. These effects were evaluated by comparing the mycelia growth rate of Trichoderma spp. when exposed to different ZEN concentrations $(0,0.5,1,2$, and $4 \mu \mathrm{g} / \mathrm{ml})$ on PDA medium. Results demonstrated that ZEN exhibited significant fungal toxic effects on Trichoderma isolates: the mycelia growth of five isolates were significantly inhibited by $1 \mu \mathrm{g} / \mathrm{ml}$ of ZEN. While for T. longibranchiatum GIM3.534, T. atroviride Q710251 and T. asperellum Q710682, the inhibitory effects were observed when treated with $2 \mu \mathrm{g} / \mathrm{ml}$ of ZEN (Figure 4).

Subsequently, all the Trichoderma isolates treated with $2 \mu \mathrm{g} / \mathrm{ml}$ ZEN were selected for further study of the metabolic activity of Trichoderma isolates on ZEN. The whole medium and mycelia were collected and prepared for analysis.

\section{Analysis of the Metabolites by LC-MS/MS}

The results revealed that glycosylated mycotoxins (Z14G, $\alpha$ ZOL14G, and $\beta$-ZOL14G) were not detected when Trichoderma isolates were cultured with ZEN. This was in accordance with the results of dual-culture assay. Interestingly, the reduced forms of ZEN ( $\alpha$-ZOL and $\beta$-ZOL) were detected in the samples
(Figure 5). T. harzianum JF309, T. harzianum GIM3.442, T. harzianum Q710613, T. atroviride Q710251, T. asperellum Q710682, and T. virens Q710925 could metabolized ZEN into $\alpha$ ZOL and $\beta$-ZOL (Figures 5B-G). Among them, T. harzianum JF309, T. harzianum GIM3.442, T. virens Q710925 converted more $\alpha$-ZOL than $\beta$-ZOL, while T. atroviride Q710251 and T. asperellum Q710682 converted more $\beta$-ZOL than $\alpha$-ZOL (Figure 6). For T. koningii GIM3.137 and T. longibranchiatum GIM3.534, only $\alpha$-ZOL was observed in the ZEN-treated experiment (Figures 5H,I).

\section{Analysis of Metabolites by LC-HRMS}

Besides glycosylation, sulfation is another detoxification process for different mycotoxins in plants and fungi. Zearalenone-14sulfate (Z14S) was found to be a metabolite in Arabidopsis thaliana, Rhizopus spp. and Aspergillus spp. when exposed to ZEN, and zearalenol-14-sulfate (ZOL14S) was observed as a fungal metabolite in ZEN-treated trial (Berthiller et al., 2007; Brodehl et al., 2014). However, there were no reference standards (Z14S and ZOL14S) available for quantitative analysis by LC-MS/MS. For detection of sulfated forms of ZEN in prepared 

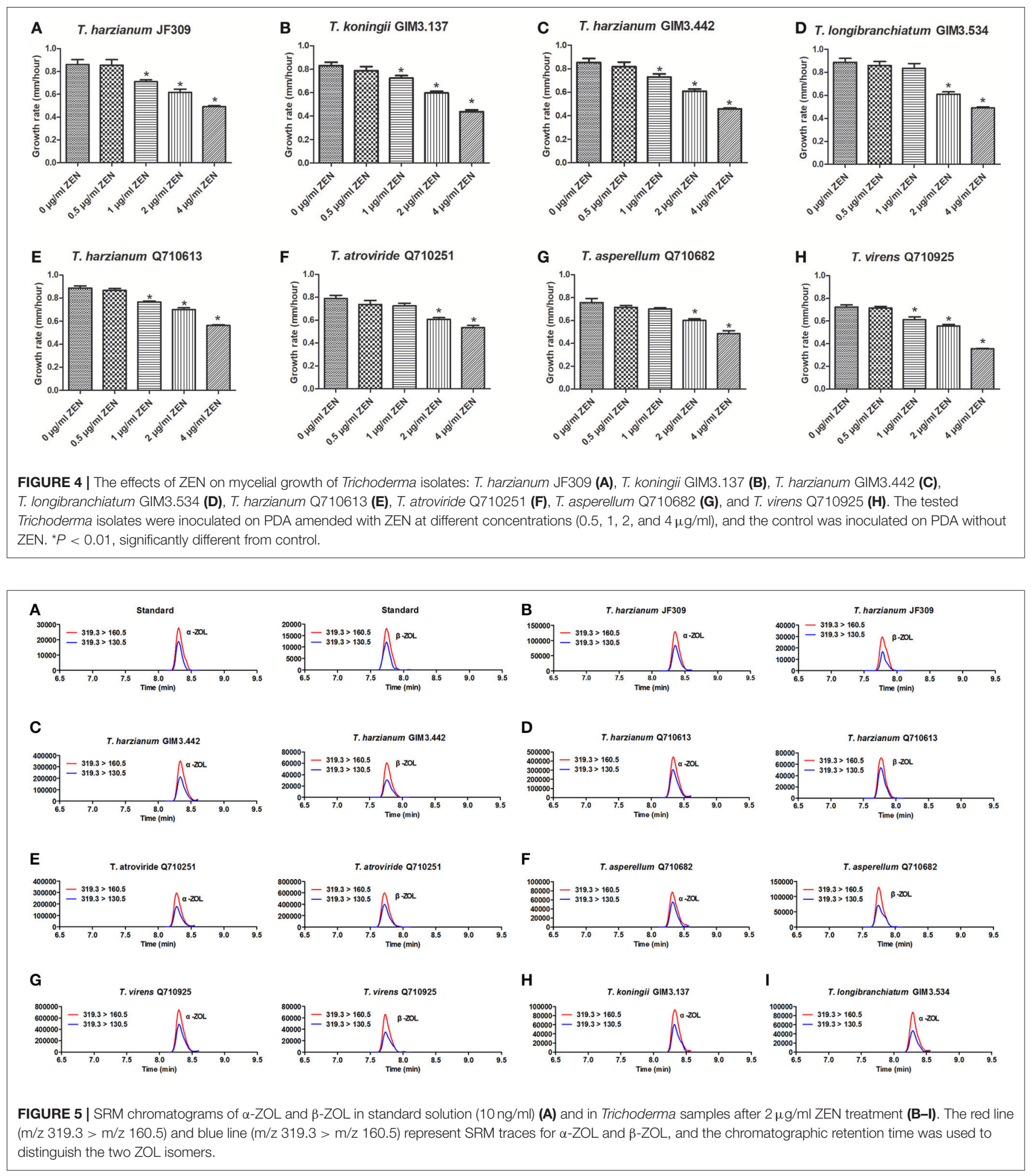

samples of Trichoderma treated with $2 \mu \mathrm{g} / \mathrm{ml} \mathrm{ZEN,} \mathrm{a} \mathrm{targeted}$ method for screening modified mycotoxins was applied on the basis of LC-HRMS (Righetti et al., 2016). The negative precursor ions $\mathrm{m} / \mathrm{z}$ 317.1394, 319.1551, 397.0963, and 399.1119 (theoretical $\mathrm{m} / \mathrm{z}$ of $\left.[\mathrm{ZEN}-\mathrm{H}]^{-},[\mathrm{ZOL}-\mathrm{H}]^{-},{ }^{\mathrm{Z} 14 \mathrm{~S}-\mathrm{H}}\right]^{-}$, and $[\mathrm{ZOL} 14 \mathrm{~S}-\mathrm{H}]^{-}$, respectively) were mass-isolated by the quadruple mass filter, and then dissociated and detected by the TOF analyzer. The precursor ion $\mathrm{m} / \mathrm{z} 397.0963$ was observed in all samples and dissociated into a fragment of 317.14 which corresponds to the $\mathrm{m} / \mathrm{z}$ of [ZEN-H] $^{-}$. In the sample of T. asperellum Q710682, precursor 
ion $\mathrm{m} / \mathrm{z} 399.1119$ was found, and could yield a fragment of $\mathrm{m} / \mathrm{z}$ 319.16 which corresponds to the $\mathrm{m} / \mathrm{z}$ of $[\mathrm{ZOL}-\mathrm{H}]^{-}$(Table 3 ). This could be explained by the fact that sulfated metabolites yield fragments $\left[\mathrm{Z} 14 \mathrm{~S}-\mathrm{SO}_{3}-\mathrm{H}\right]^{-}$and $\left[\mathrm{ZOL} 14 \mathrm{~S}-\mathrm{SO}_{3}-\mathrm{H}\right]^{-}$after losing a sulfonic group $\left(\mathrm{SO}_{3}^{-}\right)$. In addition, the other major fragments of precursor ions $\mathrm{m} / \mathrm{z} 397.0963$ and 399.1119 were in agreement with $[\mathrm{ZEN}-\mathrm{H}]^{-}(\mathrm{m} / \mathrm{z}, 317.1394)$ and $[\mathrm{ZOL}-\mathrm{H}]^{-}(\mathrm{m} / \mathrm{z}, 319.1551)$, respectively (Table 3 ). The fragments $\mathrm{m} / \mathrm{z} 317.14,175.07$ and 131.09 for $[\mathrm{Z} 14 \mathrm{~S}-\mathrm{H}]^{-}$and the fragments $\mathrm{m} / \mathrm{z} 319.15,275.20$, and 174.12 for $[\mathrm{ZOL} 14 \mathrm{~S}-\mathrm{H}]^{-}$were also reported before (Brodehl et al., 2014; Binder et al., 2017). In conclusion, these results revealed the presence of Z14S and ZOL14S in Trichoderma metabolism with ZEN treatment. For the first time, we reported

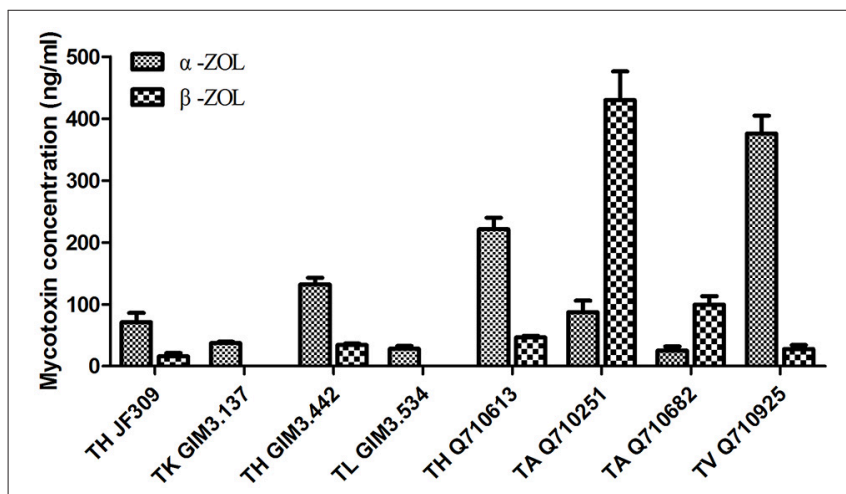

FIGURE 6 | Concentrations of the metabolites ( $\alpha$-ZOL and $\beta$-ZOL) detected in samples of $2 \mu \mathrm{g} / \mathrm{ml}$ ZEN-treated Trichoderma isolates on PDA medium. that antagonistic Trichoderma isolates possess the detoxification capability to sulfate ZEN, and these sulfated forms would be quantified when reference standards are available in future.

\section{DISCUSSION}

The effective methods to manage mycotoxin contamination include application of antagonistic microbes to prevent mycotoxin production before harvest and using detoxification agents to treat contaminated foodstuffs (Atanasova-Penichon et al., 2016; Perczak et al., 2016; De Saeger and Logrieco, 2017). Due to its potentials to control plant pathogens, the non-toxigenic Trichoderma genus has been intensively investigated (Benítez et al., 2004). In the present study, we co-cultured Trichoderma isolates with ZEN-producing F. graminearum F1 to assess the inhibition and detoxification capacities of tested Trichoderma isolates T. harzianum Q710613, T. atroviride Q710251, T. asperellum Q710682 displayed promising antagonistic potentials to control the growth and mycotoxin production of ZEN-producing F. graminearum F1. In order to exhaustively access their antagonistic potentials, these Trichoderma isolates were dual cultured with other ZEN-producing Fusarium species in the later experiment. These antagonists exhibited prominent inhibitory actions on both mycelia spread (Figure S1) and mycotoxin production (Figure S2) of the ZEN-producers. Taken together, our recent progress indicates that the three candidates are potential biological control antagonists to combat toxigenic fungi, which deserve attention and further analysis of their ability to control disease development in field experiments.

TABLE 3 | Summary of metabolites in samples of ZEN-treated Trichoderma isolates on PDA medium analyzed by LC-HRMS.

\begin{tabular}{|c|c|c|c|c|}
\hline & \multirow[t]{2}{*}{ Target compound } & \multicolumn{2}{|c|}{$\mathrm{m} / \mathbf{z}[\mathrm{M}-\mathrm{H}]^{-}$} & \multirow[t]{2}{*}{ Major fragments } \\
\hline & & Theoretical $\mathrm{m} / \mathbf{z}$ & Observed m/z & \\
\hline \multirow[t]{2}{*}{ Mycotoxin standard } & ZEN & 317.1394 & 317.1386 & $289.15,273.16,175.07,149.10,131.09$ \\
\hline & $\alpha-Z O L, \beta-Z O L$ & 319.1551 & 319.1547 & $291.21,275.20,257.26,174.12$ \\
\hline \multirow[t]{2}{*}{ T. harzianum JF309 } & Z14S & 397.0963 & 397.0953 & $317.14,289.15,273.16,175.07,149.10,131.09$ \\
\hline & ZOL14S & 399.1119 & ND & ND \\
\hline \multirow[t]{2}{*}{ T. koningii GIM3.137 } & Z14S & 397.0963 & 397.0949 & $317.14,289.15,273.16,175.07,149.10,131.09$ \\
\hline & ZOL14S & 399.1119 & ND & ND \\
\hline \multirow[t]{2}{*}{ T. harzianum GIM3.442 } & Z14S & 397.0963 & 397.0954 & $317.14,289.15,273.16,175.07,149.10,131.09$ \\
\hline & ZOL14S & 399.1119 & ND & ND \\
\hline \multirow[t]{2}{*}{ T. Iongibranchiatum GIM3.534 } & Z14S & 397.0963 & 397.0948 & $317.14,289.15,273.16,175.07,149.10,131.09$ \\
\hline & ZOL14S & 399.1119 & ND & ND \\
\hline \multirow[t]{2}{*}{ T. harzianum Q710613 } & Z14S & 397.0963 & 397.0950 & $317.14,289.15,273.16,175.07,149.10,131.09$ \\
\hline & ZOL14S & 399.1119 & ND & ND \\
\hline \multirow[t]{2}{*}{ T. atroviride Q710251 } & Z14S & 397.0963 & 397.0952 & $317.14,289.15,273.16,175.07,149.10,131.09$ \\
\hline & ZOL14S & 399.1119 & ND & ND \\
\hline \multirow[t]{2}{*}{ T. asperellum Q710682 } & Z14S & 397.0963 & 397.0953 & $317.14,289.15,273.16,175.07,149.10,131.09$ \\
\hline & ZOL14S & 399.1119 & 399.1112 & $319.15,291.21,275.20,257.26,174.12$ \\
\hline \multirow[t]{2}{*}{ T. virens Q710925 } & Z14S & 397.0963 & 397.0946 & $317.14,289.15,273.16,175.07,149.10,131.09$ \\
\hline & ZOL14S & 399.1119 & ND & ND \\
\hline
\end{tabular}

ND, Not detected. 

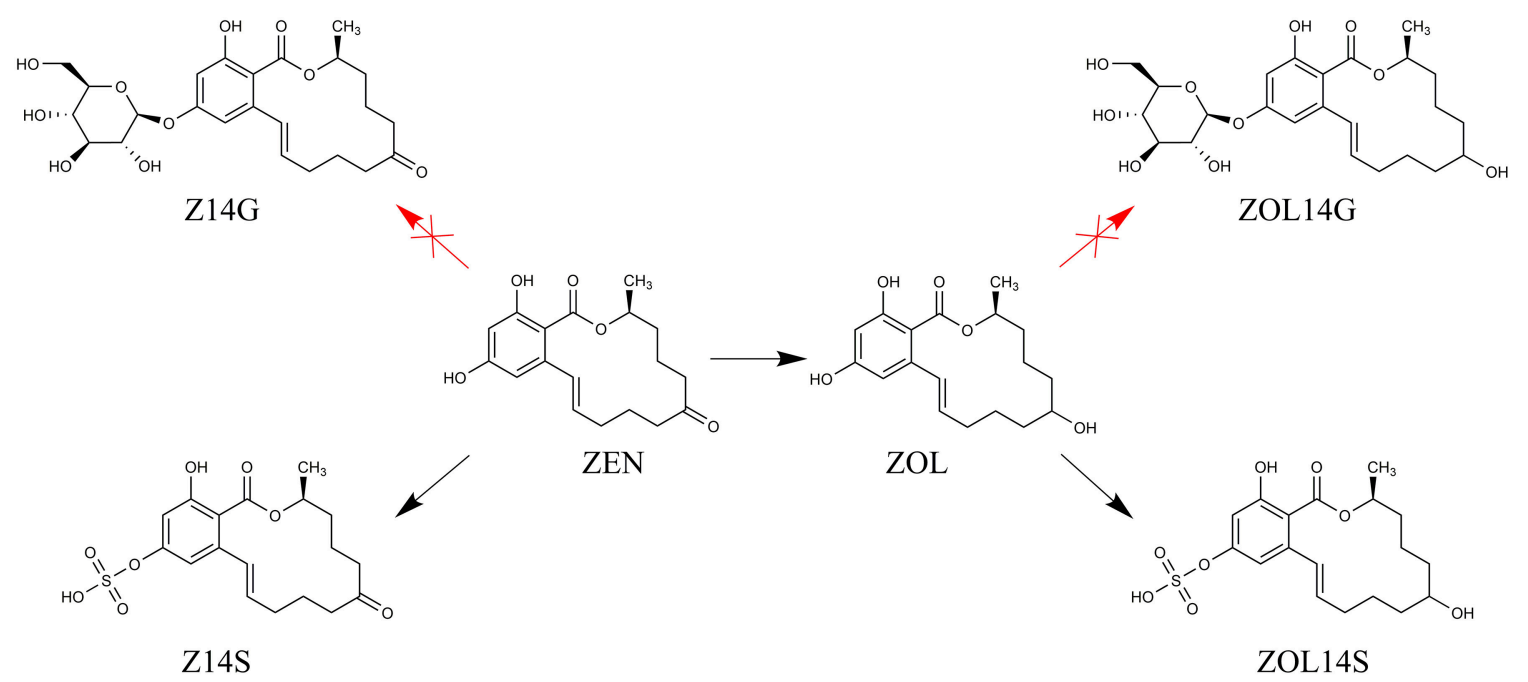

FIGURE 7 | The proposed metabolic detoxification process of mycotoxin ZEN in Trichoderma isolates.

Plants possess the capacity to detoxify phytotoxic compounds into low-toxic products after infected by toxigenic fungi. Mycotoxins are toxic xenobiotics for plants, which can be conjugated to polar metabolites in detoxification reactions of plants, generating low-toxic metabolites with structure changed (Berthiller et al., 2016). The detoxification mechanisms of plants against mycotoxins mainly include three phases: transformation phase, conjugation phase and compartmentation phase (Berthiller et al., 2007). Both glycosylation and sulfation are common processes in detoxification reactions of different plants against mycotoxins (Lemmens et al., 2016). It has been showed that DON and ZEN can be bio-transformed into glycosylated and sulfated forms in the detoxification process of plants (Berthiller et al., 2007). The UDP-glucosyltransferase (UGT) capable of converting DON into D3G was firstly identified in Arabidopsis thaliana (Poppenberger et al., 2003), and then the first UGT capable of converting ZEN into Z14G was also identified in Arabidopsis thaliana (Poppenberger et al., 2006). With regard to DON, our previous work proved that Trichoderma spp. possess the ability to metabolize DON into its glycosylated form (Tian et al., 2016b). Consequently, we explored whether Trichoderma isolates possess the ability to modify mycotoxin ZEN in this work. Not similar to plants, the tested Trichoderma isolates could not bio-transform ZEN into its glycosylated forms, but could convert ZEN into its reduced and sulfated form(s) (Figure 7). Evidence was provided that Trichoderma isolates were able to detoxify ZEN via sulfation when competing with ZEN-producing F. graminearum.

LC-MS/MS is a useful tool for simultaneous determination of different co-existing mycotoxins when standards are available (Righetti et al., 2016). However, it is still challenging to identify and quantify modified mycotoxins by using LCMS/MS due to the limited commercial availabilities of modified mycotoxin standards. The HRMS has the advantage of providing accurate ion mass-to-charge that can be used for structure elucidation of compounds in a targeted or untargeted strategy
(Righetti et al., 2016), so it has become a promising tool for analyzing the predicted metabolites without standards (De Boevre et al., 2016; Righetti et al., 2016). In our current work, the HRMS was used to obtain accurate mass and fragmentation patterns of analytes, and the sulfated metabolites (Z14S and ZOL14S) produced by Trichoderma isolates were discovered. This contributes to further investigations of the defense mechanism of biological control agents against toxigenic fungi.

\section{AUTHOR CONTRIBUTIONS}

AW and YT conceived and designed the experiments; YT, YLT, and $\mathrm{ZY}$ performed the experiments and analyzed the data; AW and YT wrote the paper; YL, JC, SD, and MD contributed materials and amended the manuscript.

\section{ACKNOWLEDGMENTS}

This work was supported by the National Basic Research Program of China (Grant 2013CB127801), National Natural Science Foundation of China (31471661, 31772087, 31701721 and 31601575), Shanghai Municipal Commission for Science and Technology (Grants 15230724400 and 17395810103), National Key R\&D Program of China (2016YFE0112900), the Special Funds for Local Scientific and Technological Development (Shanghai) under the Guidance of the Central Government, and China Agriculture Research System (CARS-02). We thank the National Center for Protein Science Shanghai for mass spectrometry assistance.

\section{SUPPLEMENTARY MATERIAL}

The Supplementary Material for this article can be found online at: https://www.frontiersin.org/articles/10.3389/fmicb. 2017.02710/full\#supplementary-material 


\section{REFERENCES}

Atanasova-Penichon, V., Barreau, C., and Richard-Forget, F. (2016). Antioxidant secondary metabolites in cereals: potential involvement in resistance to fusarium and mycotoxin accumulation. Front. Microbiol. 7:566. doi: 10.3389/fmicb.2016.00566

Benítez, T., Rincon, A. M., Limon, M. C., and Codon, A. C. (2004). Biocontrol mechanisms of Trichoderma strains. Int. Microbiol. 7, 249-260. Available online at: http://www.im.microbios.org/0704/0704249.pdf

Berthiller, F., Crews, C., Dall'Asta, C., Saeger, S. D., Haesaert, G., Karlovsky, P., et al. (2013). Masked mycotoxins: a review. Mol. Nutr. Food Res. 57, 165-186. doi: 10.1002/mnfr.201100764

Berthiller, F., Lemmens, M., Werner, U., Krska, R., Hauser, M., Adam, G., et al. (2007). Short review: metabolism of the Fusarium mycotoxins deoxynivalenol and zearalenone in plants. Mycotoxin Res. 23, 68-72. doi: 10.1007/BF029 46028

Berthiller, F., Maragos, C. M., and Dall'Asta, C. (2016). "Chapter 1 introduction to masked mycotoxins," in Masked Mycotoxins in Food: Formation, Occurrence and Toxicological Relevance, eds C. Dall'Asta and F. Berthiller (Cambridge: The Royal Society of Chemistry), 1-13.

Binder, S. B., Schwartz-Zimmermann, H. E., Varga, E., Bichl, G., Michlmayr, H., Adam, G., et al. (2017). Metabolism of zearalenone and its major modified forms in pigs. Toxins 9:56. doi: 10.3390/toxins9020056

Brodehl, A., Moller, A., Kunte, H. J., Koch, M., and Maul, R. (2014). Biotransformation of the mycotoxin zearalenone by fungi of the genera Rhizopus and Aspergillus. FEMS Microbiol. Lett. 359, 124-130. doi: 10.1111/1574-6968.12586

Busko, M., Chełkowski, J., Popiel, D., and Perkowski, J. (2008). Solid substrate bioassay to evaluate impact of Trichoderma on trichothecene mycotoxin production by Fusarium species. J. Sci. Food Agric. 88, 536-541. doi: $10.1002 /$ jsfa.3119

Castillo, M., Samar, M., Moltó, G., Resnik, S., and Pacin, A. (2002). Trichothecenes and zearalenone production by fusarium species isolated from Argentinean black beans. Mycotoxin Res. 18, 31-36. doi: 10.1007/BF02946137

Cuomo, C. A., Güldener, U., Xu, J. R., Trail, F., Turgeon, B. G., Di Pietro, A., et al. (2007). The Fusarium graminearum genome reveals a link between localized polymorphism and pathogen specialization. Science 317, 1400-1402. doi: 10.1126/science. 1143708

De Boevre, M., Ediage, E. N., Van Poucke, C., and De Saeger, S. (2016). "Chapter 4 untargeted analysis of modified mycotoxins using high-resolution mass spectrometry," in Masked Mycotoxins in Food: Formation, Occurrence and Toxicological Relevance, eds C. Dall'Asta and F. Berthiller (Cambridge: The Royal Society of Chemistry), 50-72.

De Saeger, S., and Logrieco, A. (2017). Report from the 1st MYCOKEY international conference global mycotoxin reduction in the food and feed chain held in Ghent, Belgium, 11-14 September 2017. Toxins 9:276. doi: 10.3390/toxins 9090276

Döll, S., and Dänicke, S. (2011). The Fusarium toxins deoxynivalenol (DON) and zearalenone (ZON) in animal feeding. Prev. Vet. Med. 102, 132-145. doi: 10.1016/j.prevetmed.2011.04.008

Gromadzka, K., Chelkowski, J., Popiel, D., Kachlicki, P., Kostecki, M., and Golinski, P. (2009). Solid substrate bioassay to evaluate the effect of Trichoderma and Clonostachys on the production of zearalenone by Fusarium species. World Mycotoxin J. 2, 45-52. doi: 10.3920/WMJ 2008.x046

Gromadzka, K., Waskiewicz, A., Chelkowski, J., and Golinski, P. (2008). Zearalenone and its metabolites: occurrence, detection, toxicity and guidelines. World Mycotoxin J. 1, 209-220. doi: 10.3920/WMJ2008.x015

Hussein, H. S., and Brasel, J. M. (2001). Toxicity, metabolism, and impact of mycotoxins on humans and animals. Toxicology 167, 101-134. doi: 10.1016/S0300-483X(01)00471-1

Jard, G., Liboz, T., Mathieu, F., Guyonvarc'h, A., and Lebrihi, A. (2011). Review of mycotoxin reduction in food and feed: from prevention in the field to detoxification by adsorption or transformation. Food Addit. Contam. A 28, 1590-1609. doi: 10.1080/19440049.2011.595377

Kamimura, H. (1986). Conversion of zearalenone to zearalenone glycoside by Rhizopus sp. Appl. Environ. Microbiol. 52, 515-519.
Lemmens, M., Steiner, B., Sulyok, M., Nicholson, P., Mesterhazy, A., and Buerstmayr, H. (2016). Masked mycotoxins: does breeding for enhanced Fusarium head blight resistance result in more deoxynivalenol3-glucoside in new wheat varieties? World Mycotoxin J. 9, 741-754. doi: 10.3920/WMJ2015.2029

Li, X., Shin, S., Heinen, S., Dill-Macky, R., Berthiller, F., Nersesian, N., et al. (2015). Transgenic wheat expressing a barley UDP-glucosyltransferase detoxifies deoxynivalenol and provides high levels of resistance to Fusarium graminearum. Mol. Plant Microbe Interact. 28, 1237-1246. doi: 10.1094/MPMI-03-15-0062-R

Matarese, F., Sarrocco, S., Gruber, S., Seidl-Seiboth, V., and Vannacci, G. (2012). Biocontrol of Fusarium head blight: interactions between Trichoderma and mycotoxigenic Fusarium. Microbiology 158(Pt 1), 98-106. doi: 10.1099/mic.0.052639-0

Molto, G. A., Gonzalez, H. H. L., Resnik, S. L., and Gonzalez, A. P. (1997). Production of trichothecenes and zearalenone by isolates of Fusavium spp. from Argentinian maize. Food Addit. Contam. 14, 263-268. doi: 10.1080/02652039709374523

Nielsen, L. K., Cook, D. J., Edwards, S. G., and Ray, R. V. (2014). The prevalence and impact of Fusarium head blight pathogens and mycotoxins on malting barley quality in UK. Int. J. Food Microbiol. 179, 38-49. doi: 10.1016 /j.ijfoodmicro.2014.03.023

Kovalsky Paris, M. P., Schweiger, W., Hametner, C., Stückler, R., Muehlbauer, G. J., Varga, E., et al. (2014). Zearalenone-16-O-glucoside: a new masked mycotoxin. J. Agric. Food Chem. 62, 1181-1189. doi: 10.1021/jf40 $5627 \mathrm{~d}$

Pasquet, J.-C., Changenet, V., Macadré, C., Boex-Fontvieille, E., Soulhat, C., Bouchabké-Coussa, O., et al. (2016). A Brachypodium UDPglycosytransferase confers root tolerance to deoxynivalenol and resistance to Fusarium infection. Plant Physiol. 172, 559-574. doi: 10.1104/pp.16. 00371

Perczak, A., Jus, K., Marchwinska, K., Gwiazdowska, D., Waskiewicz, A., and Golinski, P. (2016). Degradation of zearalenone by essential oils under in vitro conditions. Front. Microbiol. 7:1224. doi: 10.3389/fmicb.2016. 01224

Pietsch, C., Kersten, S., Burkhardt-Holm, P., Valenta, H., and Dänicke, S. (2013). Occurrence of deoxynivalenol and zearalenone in commercial fish feed: an initial study. Toxins 5:184. doi: 10.3390/toxins50 10184

Poppenberger, B., Berthiller, F., Bachmann, H., Lucyshyn, D., Peterbauer, C., Mitterbauer, R., et al. (2006). Heterologous expression of Arabidopsis UDP-glucosyltransferases in Saccharomyces cerevisiae for production of zearalenone-4-O-glucoside. Appl. Environ. Microbiol. 72, 4404-4410. doi: 10.1128/AEM.02544-05

Poppenberger, B., Berthiller, F., Lucyshyn, D., Sieberer, T., Schuhmacher, R., Krska, R., et al. (2003). Detoxification of the Fusarium mycotoxin deoxynivalenol by a UDP-glucosyltransferase from Arabidopsis thaliana. J. Biol. Chem. 278, 47905-47914. doi: 10.1074/jbc.M307552200

Righetti, L., Paglia, G., Galaverna, G., and Dall'Asta, C. (2016). Recent advances and future challenges in modified mycotoxin analysis: why HRMS has become a key instrument in food contaminant research. Toxins 8:361. doi: 10.3390/toxins 8120361

Schweiger, W., Boddu, J., Shin, S., Poppenberger, B., Berthiller, F., Lemmens, M., et al. (2010). Validation of a candidate deoxynivalenol-inactivating UDP-glucosyltransferase from barley by heterologous expression in yeast. Mol. Plant Microbe Interact. 23, 977-986. doi: 10.1094/MPMI-237-0977

Sellamani, M., Kalagatur, N. K., Siddaiah, C., Mudili, V., Krishna, K., Natarajan, G., et al. (2016). Antifungal and zearalenone inhibitory activity of Pediococcus pentosaceus isolated from dairy products on Fusarium graminearum. Front. Microbiol. 7:890. doi: 10.3389/fmicb.2016.00890

Stepien, Ł., and Chełkowski, J. (2010). Fusarium head blight of wheat: pathogenic species and their mycotoxins. World Mycotoxin J. 3, 107-119. doi: 10.3920/WMJ2009.1193

Tian, Y., Tan, Y., Liu, N., Liao, Y., Sun, C., Wang, S., et al. (2016a). Functional agents to biologically control deoxynivalenol contamination in cereal grains. Front. Microbiol. 7:395. doi: 10.3389/fmicb.2016.00395 
Tian, Y., Tan, Y., Liu, N., Yan, Z., Liao, Y., Chen, J., et al. (2016b). Detoxification of deoxynivalenol via glycosylation represents novel insights on antagonistic activities of Trichoderma when confronted with Fusarium graminearum. Toxins 8:335. doi: 10.3390/toxins 8110335

Utermark, J., and Karlovsky, P. (2007). Role of zearalenone lactonase in protection of Gliocladium roseum from fungitoxic effects of the mycotoxin zearalenone. Appl. Environ. Microbiol. 73, 637-642. doi: 10.1128/AEM.01440-06

Zinedine, A., Soriano, J. M., Molto, J. C., and Manes, J. (2007). Review on the toxicity, occurrence, metabolism, detoxification, regulations and intake of zearalenone: an oestrogenic mycotoxin. Food Chem. Toxicol. 45, 1-18. doi: $10.1016 /$ j.fct.2006.07.030
Conflict of Interest Statement: The authors declare that the research was conducted in the absence of any commercial or financial relationships that could be construed as a potential conflict of interest.

Copyright (c) 2018 Tian, Tan, Yan, Liao, Chen, De Boevre, De Saeger and Wu. This is an open-access article distributed under the terms of the Creative Commons Attribution License (CC BY). The use, distribution or reproduction in other forums is permitted, provided the original author(s) or licensor are credited and that the original publication in this journal is cited, in accordance with accepted academic practice. No use, distribution or reproduction is permitted which does not comply with these terms. 